\title{
Encryption And Decryption of a Message Involving Byte Rotation Technique and Invertible Matrix
}

\author{
Amit Kumar Mandle, Varsha Namdeo
}

\begin{abstract}
The aim of this paper is to introduce a new encryption algorithm involving byte rotation and invertible matrix. In the proposed algorithm firstly we apply byte rotation to get an intermediate cipher and then applying the invertible matrix (modulo 27), which gives the final cipher text. Using secret key matrix along with congruence modulo, the message can be encrypted and decrypted perfectly.
\end{abstract}

Keywords: Congruence, Byte Rotation Invertible Matrix, Encryption and Decryption.

\section{INTRODUCTION}

$F_{\text {or hiding the information we use cryptography, which is }}$ an art of encryption and decryption. Cryptography is a mathematical technique, which is used for information security. It plays vital role in cellular communication viz. ATM Card, transmitting funds, digital signature etc. It is categories into two parts symmetric and asymmetric. When sender and receiver both use the same key for encryption and decryption, then it is known as symmetric cryptography. But in asymmetric cryptography w use two different keys [3, 5].

In this research paper we use byte rotation technique and invertible matrix. Firstly we apply matrix operation under modulo system to get intermediate cipher. After that on applying byte rotation technique on different blocks of plaintext, we obtain the final cipher text. A matrix is invertible if it is non-singular. To encrypt the message the key matrix used and for decrypt the encoded message we use inverse of the matrix under modulo system. The key matrix should be secret between sender and receiver.

A square matrix $X$ is said to be an invertible matrix iff there exist another square matrix Ys.t.XY = YX = I. It may be noted that all the square matrices are not invertible. If a square matrix has an invertible matrix or non-singular, then its determinant value must be non-zero.

Revised Manuscript Received on December 30, 2019.

* Correspondence Author

Amit Kumar Mandle, Research Scholar, Department of Computer Applications (MCA) SRK University, Bhopal, India.

Varsha Namdeo, Associate Professor, Department of Computer Science Engineering, SRK University, Bhopal, India.

(C) The Authors. Published by Blue Eyes Intelligence Engineering and Sciences Publication (BEIESP). This is an open access article under the CC BY-NC-ND license (http://creativecommons.org/licenses/by-nc-nd/4.0/)

\section{LITERATURE REVIEW:}

Sani Isa [6], Hamed [4], Bhati [1], Bhati [2] and other authors introduced various algorithms for encryption and decryption of a message involving Byte rotation techniques and using of invertible matrix separately time to time. For encryption and decryption there is no single algorithm is sufficient. Therefore researchers worked hard to remove the deficiency and finding better algorithm.

\section{METHODOLOGY}

To provide sufficient security we use multiple encryption and multilevel encryption system. Here we develop an algorithm model having two steps. Firstly,we apply the secret key matrix along with congruence modulo 27 and obtain an intermediate cipher. Thereafter we apply the byte rotation technique (agree both sender and receiver), to get final cipher text.

For decrypting the message we will use the reverse process of encryption along with byte rotation technique and invertible matrix of congruence modulo 27.

Numerical values for alphabets and some symbols used in the paper given in the following table:

Table - 1

\begin{tabular}{|l|l|l|l|}
\hline A & 1 & O & 15 \\
\hline B & 2 & P & 16 \\
\hline C & 3 & Q & 17 \\
\hline D & 4 & R & 18 \\
\hline E & 5 & S & 19 \\
\hline F & 6 & T & 20 \\
\hline G & 7 & U & 21 \\
\hline H & 8 & V & 22 \\
\hline I & 9 & W & 23 \\
\hline J & 10 & X & 24 \\
\hline K & 11 & Y & 25 \\
\hline L & 12 & Z & 26 \\
\hline M & 13 & Space & 0 \\
\hline N & 14 & & \\
\hline
\end{tabular}

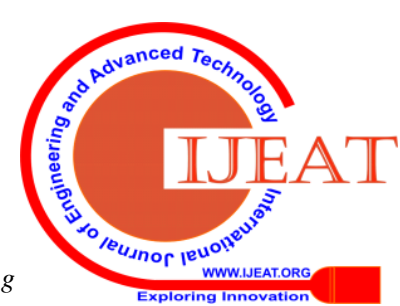




\section{ALGORITHM}

\subsection{Encryption:}

1. Take a non-singular square matrix of order 4 as key (say $\mathrm{K})$.

2. Arrange the character/symbol of plain text in a block size of 16 bytes as $4 \times 4$ matrix.

3. Convert the alphabet/symbol into their corresponding values using Table1 and call this resultant matrix $\mathrm{M}$.

4. Multiply the key matrix $\mathrm{K}$ and plain text matrix $\mathrm{M}$ under modulo 27 i.e.

$\mathrm{MK}(\bmod 27)=\mathrm{M}_{1}$ (say)

5. Obtain the transpose of $\mathrm{M}_{1}$, say $M_{1}^{X}$.

6. Apply the vertical rotation (as per agreement of sender and receiver) on last three columns of $M_{1}^{X}$. Call this resultant matrix $\mathrm{C}_{1}$.

7. Now apply the horizontal rotation of last three rows of $\mathrm{C}_{1}$ (as per agreement of sender and receiver). Obtain another matrix say $\mathrm{C}_{2}$.

8. Convert the numeric values of element of $\mathrm{C}_{2}$ into alphabets using Table1, we get a cipher text.

\subsection{Decryption:}

In a reverse process of encryption having following steps:

1. Take the cipher text and arrange it in a square matrix of order 4 of block sized of 16 bytes. After arranging correct them into numeric values using table $\mathrm{I}$, and get a resultant matrix say $\mathrm{D}_{1}$.

2. Apply the horizontal rotation (same as encryption method) on $\mathrm{D}_{1}$, we get a matrix say $\mathrm{D}_{2}$.

3. Now applying the vertical rotation (same as encryption method) on $\mathrm{D}_{2}$, we get a matrix say $\mathrm{D}_{3}$.

4. Obtain the transpose of $\mathrm{D}_{3}$, say $\mathrm{D}_{4}$.

5. Now calculate $\mathrm{D}_{4} \mathrm{k}^{-1}(\bmod 27)=\mathrm{M}$ (say)

6. Convert the element of $\mathrm{M}$ into alphabet/symbol using Table I and arrange them row wise we get a plain text.

\section{ILLUSTRATION:}

\section{Encryption Steps:}

1. Consider a non-singular key matrix of order $4 \times 4$ as follows:

$$
K=\left[\begin{array}{llll}
2 & 1 & 2 & 1 \\
3 & 5 & 2 & 2 \\
5 & 1 & 3 & 1 \\
3 & 1 & 3 & 2
\end{array}\right]
$$

2. Let plain text is

\section{SYMMETRIC CIPHER}

Arrange them in a block size of 16 bytes i.e. $4 \times 4$ matrix, we get

$$
\left[\begin{array}{cccc}
\mathrm{S} & \mathrm{Y} & \mathrm{M} & \mathrm{M} \\
\mathrm{E} & \mathrm{T} & \mathrm{R} & \mathrm{I} \\
\mathrm{C} & 0 & \mathrm{C} & \mathrm{I} \\
\mathrm{P} & \mathrm{H} & \mathrm{E} & \mathrm{R}
\end{array}\right]
$$

3. In the above block matrix substitute numeric values of letters, as follows:

$$
M=\left[\begin{array}{cccr}
19 & 25 & 13 & 13 \\
5 & 20 & 18 & 9 \\
3 & 0 & 3 & 9 \\
16 & 8 & 5 & 18
\end{array}\right]
$$

4. Multiply the key matrix and text matrix $\mathrm{M}$ under modulo system, we get the following Multiplicative matrix $\mathrm{M}_{1}=\mathrm{MK}(\bmod 27)$

$$
\begin{gathered}
=\left[\begin{array}{cccc}
19 & 25 & 13 & 13 \\
5 & 20 & 18 & 9 \\
3 & 0 & 3 & 9 \\
16 & 8 & 5 & 18
\end{array}\right]\left[\begin{array}{cccc}
2 & 1 & 2 & 1 \\
3 & 5 & 2 & 2 \\
5 & 1 & 3 & 1 \\
3 & 1 & 3 & 2
\end{array}\right](\bmod 27) \\
=\left[\begin{array}{cccc}
1 & 8 & 4 & 0 \\
25 & 25 & 23 & 0 \\
21 & 15 & 15 & 24 \\
0 & 25 & 9 & 19
\end{array}\right]
\end{gathered}
$$

5. Obtain the transpose of $\mathrm{M}_{1}$, we get

$$
M_{\text {q }}=\left[\begin{array}{cccc}
1 & 25 & 21 & 0 \\
8 & 25 & 15 & 25 \\
4 & 23 & 15 & 9 \\
0 & 0 & 24 & 19
\end{array}\right]
$$

6. Now apply the vertical rotation on last three columns. $M_{1}^{X}$ such that rotate three bytes from 2nd column, rotate two bytes from 3rdcolumn, rotate one byte from 4thcolumn and 1stcolumn unchanged. Denote the resultant matrix by $\mathrm{C} 1$, we get

$$
\mathrm{C}_{1}=\left[\begin{array}{cccc}
1 & 0 & 15 & 25 \\
8 & 25 & 24 & 9 \\
4 & 24 & 21 & 19 \\
0 & 23 & 15 & 0
\end{array}\right]
$$

7. Applying the horizontal rotation on last three rows of $C_{1}$ such that rotate three bytes from $2^{\text {nd }}$ row, rotate two bytes from $3^{\text {rd }}$ row, rotate one byte from $4^{\text {th }}$ row and $1^{\text {st }}$ row remains unchanged. Denote the resultant matrix by $\mathrm{C}_{2}$, we get -

$$
\mathrm{C} 2=\left[\begin{array}{cccc}
1 & 0 & 15 & 25 \\
25 & 24 & 9 & 8 \\
21 & 19 & 4 & 24 \\
23 & 15 & 0 & 0
\end{array}\right]
$$

8. Convert the numeric values of C2 into their corresponding alphabet letters using table1, we get the following cipher text block

$$
\mathrm{C}_{3}=\left[\begin{array}{cccc}
\mathrm{A} & 0 & 0 & \mathrm{Y} \\
\mathrm{Y} & \mathrm{X} & \mathrm{I} & \mathrm{H} \\
\mathrm{U} & \mathrm{S} & \mathrm{D} & \mathrm{X} \\
\mathrm{W} & 0 & 0 & 0
\end{array}\right]
$$

Therefore cipher text is

\section{A0OYYXIHUSDXWO00}

\section{Decryption Steps:}

1. Consider the cipher text A0OYYXIHUSDXWO00

2. Arrange it in block size of 16 bytes i.e. $4 \times 4$ matrix and convert them into their corresponding numeric value using Table1, we get -

$\left[\begin{array}{cccc}1 & 0 & 15 & 25 \\ 25 & 24 & 9 & 8 \\ 21 & 19 & 4 & 24 \\ 23 & 15 & 0 & 0\end{array}\right]=\mathrm{D}_{1}$ (say)

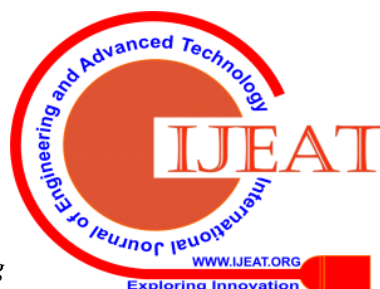


3. Applying the horizontal rotation on last three rows of $\mathrm{D}_{1}$ s.t.rotate three bytes from $2^{\text {nd }}$ row, rotate two bytes from $3^{\text {rd }}$ row rotate one byte from $4^{\text {th }}$ row and $1^{\text {st }}$ row remains unchanged, we get -

$$
\left[\begin{array}{cccc}
1 & 0 & 15 & 25 \\
8 & 25 & 24 & 9 \\
4 & 24 & 21 & 19 \\
0 & 23 & 15 & 0
\end{array}\right]=\mathrm{D}_{2} \text { (say) }
$$

4. Now apply the vertical rotation on last three columns of $\mathrm{D}_{2}$ s.t. rotate three bytes from $2^{\text {nd }}$ column, rotate two bytes from $3^{\text {rd }}$ column, rotate one byte from $4^{\text {th }}$ column and $1^{\text {st }}$ column remains unchanged, we get -

$$
\left[\begin{array}{cccc}
1 & 25 & 21 & 0 \\
8 & 24 & 15 & 25 \\
4 & 23 & 15 & 9 \\
0 & 0 & 24 & 19
\end{array}\right]=\mathrm{D}_{\mathrm{a}} \text { (say) }
$$

5.Obtain the transpose of $\mathrm{D}_{3}$, we get -

$$
\left[\begin{array}{cccc}
1 & 8 & 4 & 0 \\
25 & 24 & 23 & 0 \\
21 & 15 & 15 & 24 \\
0 & 25 & 9 & 19
\end{array}\right]=\mathrm{D}_{4} \text { (say) }
$$

6. Now calculate -

$$
\begin{aligned}
& \mathrm{D}_{4} \mathrm{~K}^{-1}(\bmod 27)=\mathrm{M} \text { (says) } \\
& \Rightarrow \mathrm{M}=\left[\begin{array}{cccc}
1 & 8 & 4 & 0 \\
25 & 24 & 23 & 0 \\
21 & 15 & 15 & 24 \\
0 & 25 & 9 & 19
\end{array}\right]\left[\begin{array}{cccc}
16 & 5 & 25 & 15 \\
8 & 10 & 22 & 2 \\
5 & 12 & 7 & 9 \\
5 & 10 & 22 & 4
\end{array}\right](\bmod 27) \\
&=\left[\begin{array}{rrrr}
19 & 25 & 13 & 13 \\
5 & 20 & 18 & 9 \\
3 & 0 & 3 & 9 \\
16 & 8 & 5 & 18
\end{array}\right]
\end{aligned}
$$

7. Convert the above matrix into their corresponding alphabet/symbol using Table1, we get a matrix of order $4 \times 4$ of block size 16 as follows:

$$
\left[\begin{array}{cccc}
\mathrm{S} & \mathrm{Y} & \mathrm{M} & \mathrm{M} \\
\mathrm{E} & \mathrm{T} & \mathrm{R} & \mathrm{I} \\
\mathrm{C} & \mathrm{O} & \mathrm{C} & \mathrm{I} \\
\mathrm{P} & \mathrm{H} & \mathrm{E} & \mathrm{R}
\end{array}\right]
$$

8. Arrange them in row wise we get the original plain text as

\section{SYMMETRIC CIPHER}

\section{RESULT AND DISCUSSION}

In order to encrypt and decrypt a message, in this paper we used invertible key matrix congruent modulo 27 . To keep the information secure from others (except receiver) mathematical relations have been logically implemented. Among the other cryptographic technique using of matrices, is the strongest method since it uses mathematical logics.

Due to the chosen byte rotation technique along with invertible key matrix congruent modulo 27 , it is very difficult to extract the original information. Due to key size (16 bytes) brute force attack is also difficult. Explanation of result is as follows:

\begin{tabular}{|c|c|c|c|}
\hline SN & $\begin{array}{c}\text { Name of } \\
\text { attack }\end{array}$ & $\begin{array}{c}\text { Opportunity of } \\
\text { attack }\end{array}$ & Explanation \\
\hline 1 & $\begin{array}{c}\text { Cipher text } \\
\text { attack }\end{array}$ & Very Difficult & $\begin{array}{c}\text { Due to the chosen Byte rotation } \\
\text { technique and invertible key } \\
\text { matrix(size is 16 bytes) congruent } \\
\text { modulo 27 }\end{array}$ \\
\hline
\end{tabular}

\begin{tabular}{|c|c|l|c|}
\hline 2 & $\begin{array}{c}\text { Chosen } \\
\text { cipher text } \\
\text { attack }\end{array}$ & Very Difficult & $\begin{array}{c}\text { Due to the chosen Byte rotation } \\
\text { technique and invertible key } \\
\text { matrix(size is 16 bytes) congruent } \\
\text { modulo 27 }\end{array}$ \\
\hline 3 & $\begin{array}{c}\text { Adaptive } \\
\text { chosen } \\
\text { cipher text } \\
\text { attack }\end{array}$ & Very Difficult & $\begin{array}{c}\text { Due to the chosen Byte rotation } \\
\text { technique and invertible key } \\
\text { matrix(size is 16 bytes) } \\
\text { congruent modulo 27 }\end{array}$ \\
\hline 5 & $\begin{array}{c}\text { plain text } \\
\text { attack } \\
\text { plain text } \\
\text { attack }\end{array}$ & Difficult & $\begin{array}{c}\text { Due to the chosen Byte rotation } \\
\text { technique and invertible key } \\
\text { matrix (size is 16 bytes) } \\
\text { congruent modulo 27 }\end{array}$ \\
\hline 6 & $\begin{array}{c}\text { Adaptive } \\
\text { chosen } \\
\text { plain text } \\
\text { attack }\end{array}$ & Difficult & $\begin{array}{c}\text { Due to the chosen Byte rotation } \\
\text { technique and invertible key } \\
\text { matrix (size is 16 bytes) } \\
\text { congruent modulo 27 }\end{array}$ \\
\hline
\end{tabular}

\section{CONCLUSION}

Since in this paper, we use the byte rotation technique and invertible matrix. Therefore the proposed algorithm in this paper arise the strong security system and produced cipher text cannot be broken easily.

It is also generate the double encryption system, firstly from invertible matrix and secondly, from byte rotation technique. Using the above method the information could be sent and received safely. Without key matrix and congruence relations the message could not decrypt.

\section{REFERENCES}

1. BhatiSunita and Sharma S.K.: Block wise parallel encryption through multithreading Concept, Aishwarya Research Communication Journal, Vol.3, 2011, pp. 101-106.

2. BhatiSunita, Bhati Anita and Sharma S.K.: A new approach towards Encryption Schemes: Byte-Rotation Encryption Algorithm, Proceedings of the Word Congress on Engineering and Computer Science, 2012, Vol.11, pp. 1-4.

3. ForouzanBehrouz A.: Cryptography \& Network Security, McGraw Hill Education, 2007.

4. HamedAbdulaziz B.M. and Albudawe Ibrahim O.A.: Encrypt and Decrypt Message Using Invertible Matrices Modulo 27, AJER, Vol.6, Issue 6, 2017, pp. 212-217.

5. KahateAtul: Cryptography and Network Security, Tata McGraw Hill, New Delhi, 2008.

6. Soni Isa and Abdulaziz B.M. Hamed: Cryptography Using Congruence Modulo Relations, American Journal of Engineering Research, Vol.6, Issue 3, 2017 pp. 156-160.

\section{AUTHORS PROFILE}

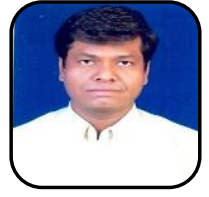

Amit Kumar Mandle is a Research Scholar in the Department of Computer Applications (MCA) at SRK University, Bhopal, India. He completed his Master in Computer Application from MPBOU, Bhopal (M.P.) in 2006.

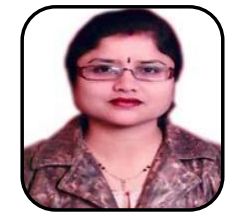

Varsha Namdeo is an Associate Professor in the Department of Computer Science and Engineering at SRK University, Bhopal, India. She is a teacher and researcher in the field of computer science and information technology. She earned her Master in Computer Application from Barkatullah University Bhopal (M.P.) in 2000 and in Computer Science and Engineering from Barkatullah University Bhopal (M.P.) in 2009 and $\mathrm{PhD}$ degree from Maulana Azad National Institute of Technology; Bhopal (M.P.) in 2015. She had a long career in teaching and research. 